\title{
DISC1 regulates lactate metabolism in astrocytes: implications for psychiatric disorders
}

Yan Jouroukhin (1) ', Yusuke Kageyama², Varvara Misheneva', Alexey Shevelkin', Shaida Andrabi', Emese Prandovszky ${ }^{4}$, Robert H. Yolken ${ }^{4}$, Valina L. Dawson ${ }^{5,6,7,8}$, Ted M. Dawson ${ }^{5,7,8,9}$, Susan Aja ${ }^{10}$, Hiromi Sesaki ${ }^{11}$ and

Mikhail V. Pletnikov 1,7,12,13

\begin{abstract}
Our knowledge of how genetic risk variants contribute to psychiatric disease is mainly limited to neurons. However, the mechanisms whereby the same genetic risk factors could affect the physiology of glial cells remain poorly understood. We studied the role of a psychiatric genetic risk factor, Disrupted-In-Schizophrenia-1 (DISC1), in metabolic functions of astrocytes. We evaluated the effects of knockdown of mouse endogenous DISC1 (DISC1-KD) and expression of a dominant-negative, C-terminus truncated human DISC1 (DN-DISC1) on the markers of energy metabolism, including glucose uptake and lactate production, in primary astrocytes and in mice with selective expression of DN-DISC1 in astrocytes. We also assessed the effects of lactate treatment on altered affective behaviors and impaired spatial memory in DN-DISC1 mice. Both DISC1-KD and DN-DISC1 comparably decreased mRNA and protein levels of glucose transporter 4 and glucose uptake by primary astrocytes. Decreased glucose uptake was associated with reduced oxidative phosphorylation and glycolysis as well as diminished lactate production in vitro and in vivo. No significant effects of DISC1 manipulations in astrocytes were observed on expression of the subunits of the electron transport chain complexes or mitofilin, a neuronal DISC1 partner. Lactate treatment rescued the abnormal behaviors in DN-DISC1 male and female mice. Our results suggest that DISC1 may be involved in the regulation of lactate production in astrocytes to support neuronal activity and associated behaviors. Abnormal expression of DISC1 in astrocytes and resulting abnormalities in energy supply may be responsible for aspects of mood and cognitive disorders observed in patients with major psychiatric illnesses.
\end{abstract}

\section{Introduction}

Astrocytes are the most abundant cells in the brain, and pathological changes in astrocytes are likely to contribute to cognitive impairment and behavioral disorders ${ }^{1,2}$. Because of extensive tissue distribution and ramified morphology, astrocytes are in contact with neurons, glia and blood vessel cells ${ }^{3,4}$. Astrocytes uptake glucose and

\footnotetext{
Correspondence: Mikhail V Pletnikov (mpletni1@jhu.edu)

${ }^{1}$ Departments of Psychiatry and Behavioral Sciences, Johns Hopkins University School of Medicine, Baltimore, MD 21287, USA

${ }^{2}$ Departments of Pathology, Johns Hopkins University School of Medicine, Baltimore, MD 21287, USA
}

Full list of author information is available at the end of the article ketones to produce lactate via glycolysis or glycogenolysis, providing energy support to neurons ${ }^{5}$. Deficits in astrocyte energy supply can affect neuronal activity, resulting in behavioral and cognitive dysfunction ${ }^{5}$.

Recent progress in psychiatric genetics has advanced our knowledge of how genetic variants can affect neurodevelopment and adult brain function ${ }^{6}$. Unfortunately, our understanding of the underlying molecular mechanisms is limited to neurons, and little is known of the pathogenic contributions of genetic risk factors within glia, including astrocytes ${ }^{7,8}$.

Disrupted-In-Schizophrenia-1 (DISC1) is a gene disrupted by balanced (1:11) (q42.1; q14.3) translocation, 
segregating in the Scottish family with several major psychiatric disorders, including schizophrenia, depression, and bipolar disorder ${ }^{9}$. Despite its original name, DISC1 has not been associated with schizophrenia in the latest genome-wide association studies (GWAS) ${ }^{10}$ and is likely a general psychiatric risk factor that may be involved in the molecular pathogenesis of several neuropsychiatric disorders. As a genetic element of the highly penetrant ultrarare chromosomal translocation, DISC1 and its protein products could be used as valuable molecular tools for mechanistic studies to advance our understanding of the molecular pathobiology of several major psychiatric diseases, irrespective of their categorical classifications ${ }^{11}$.

Multiple reports have demonstrated that DISC1 localizes to mitochondria and is involved in mitochondria trafficking and functions, including oxidative phosphorylation, ATP production and calcium buffering ${ }^{12-18}$. However, all these studies were performed on neuronal models $^{17,19}$ and whether the same partners, underlying mechanisms and/or functional consequences of altered expression of DISC1 can be observed in other brain cells remains unknown although a recent report demonstrated cell-type-specific molecular changes due to expression of DISC1 variants ${ }^{20}$. Thus, we sought to determine the role (s) of DISC1 in energy metabolism in astrocytes. Our findings show, for the first time, that both knockdown of endogenous DISC1 and expression of a dominant-negative, C-terminus truncated human DISC1 (DN-DISC1) produced decreased levels of the glucose transporter 4 (GLUT4), diminished uptake of glucose, and reduced production of lactate by astrocytes. The metabolic perturbations in DN-DISC1 mice were associated with altered affective behaviors and deficient memory that were rescued by systemic lactate treatment.

\section{Materials and methods General design}

To study the role of DISC1 in energy metabolism in astrocytes, we employed two complementary approaches: knockdown of endogenous mouse Disc1 (DISC1-KD) in primary astrocytes or expression of a dominant-negative, C-terminus truncated human DISC1 (DN-DISC1) in primary astrocytes or mice.

\section{Measurement of mitochondrial membrane potential $(\Delta \Psi)$ and mitochondrial morphology}

To determine the effects of DISC1-KD or DN-DISC1 on the morphology or the membrane potential of mitochondria, primary astrocytes were incubated with $100 \mathrm{nM}$ of the membrane-potential-independent MitoTracker Green (MTG, M7514, Invitrogen-Molecular Probes) and $8 \mathrm{nM}$ of the membrane-potential-dependent tetramethylrhodamine, ethyl ester (TMRE, T669, Invitrogen-
Molecular Probes) for $30 \mathrm{~min}$. Afterwards, cells were gently washed three times with PBS and incubated with live cell imaging solution (A14291DJ, Molecular Probes). Live cells were imaged using a confocal microscope (LSM 510 Meta, Carl Zeiss) with a 100× oilimmersion objective. MTG was excited with a 488-nm argon laser, and emission was recorded with a 500 to 550 $\mathrm{nm}$ bandpass filter. TMRE was excited with a 543-nm helium-neon laser, and emission was recorded with a 650 to 710-nm bandpass filter. Data were analyzed and are presented as the TMRE/MTG ratio. The ratio of the area occupied by swollen, spherical mitochondria with reduced $\Delta \Psi^{21}$ to the area occupied by all mitochondria in a given astrocyte was calculated. At least 20 cells were evaluated for each group.

\section{Measurements of oxygen consumption rate (OCR)}

The mitochondrial OCR was assessed in primary astrocytes using an XF24 Extracellular Flux Analyzer (Seahorse Bioscience) as described previously ${ }^{22}$. Briefly, approximately 100,000 primary astrocytes were plated at a density of $50 \times 10^{3}$ per well in XF24 cell-culture microplates pre-coated with poly-D-lysine (Sigma P6407). The cultures were incubated for $24 \mathrm{~h}$ in growth medium. The next day, the medium was replaced with Seahorse XF medium supplemented with $10 \mathrm{mM}$ glucose (Sigma, G8769), $1 \mathrm{mM}$ L-glutamine (Life Technologies, 25030081) and $1 \mathrm{mM}$ sodium pyruvate (Life Technologies, 11360070). OCR was analyzed in an XF24 analyzer after $45 \mathrm{~min}$ of incubation at $37^{\circ} \mathrm{C}$ in a $\mathrm{CO}_{2}$-free incubator. The first OCR measurement was recorded following 11min equilibration, a 1-min mix period, and a 1-min wait period. Oligomycin, carbonyl cyanide m-chlorophenylhydrazone, (CCCP) and rotenone were sequentially added to each well to assess basal respiration, coupling of the respiratory chain, and mitochondrial respiratory capacity, respectively.

\section{Measurement of glycolysis as extracellular acidification rate \\ Primary astrocytes were plated at a density of $50 \times 10^{3}$ per well in XF24 cell-culture microplates for $48 \mathrm{~h}$. On the day of assay, the medium was replaced with glucose-free XF24 Seahorse medium. Glycolytic flux (basal glycolysis, glycolytic capacity, and glycolytic reserve) was assessed as the extracellular acidification rate (ECAR) by sequentially adding glucose, oligomycin, and 2-deoxyglucose to the XF24 flux analyzer ${ }^{23}$. ECAR was measured at $37^{\circ} \mathrm{C}$ with a 1-min mix, 1-min wait, and 2-min measurement protocol. Seahorse analysis was started after $45 \mathrm{~min}$ of incubation in a $\mathrm{CO}_{2}$-free incubator. The first ECAR measurement was recorded after 11-min equilibration, a 1-min mix period, and a 1-min wait period.}




\section{Glucose uptake assay}

Glucose uptake was measured in culture medium using a glucose uptake cell-based assay kit (600470, Cayman). Primary astrocytes were seeded the day before at a density of 10,000 cells per well in 96-well plates. The fluorescently labeled glucose analog 2-N-(7-nitro-benz-2-oxa-1,3-diazol-4-yl) amino)-2 deoxyglucose (2-NBDG) was used to measure glucose uptake. Primary astrocytes were incubated with 2-NBDG for $30 \mathrm{~min}$ and then washed three times with phosphate-buffered saline (PBS). Cell lysate was used to detect the fluorescence signal at the excitation and emission wavelengths of 485 and $535 \mathrm{~nm}$, respectively, using a GloMax Discover System (Promega) luminometer in a 96-well microplate.

\section{Lactate measurement in primary astrocytes}

Lactate was measured in medium using an L-lactate assay kit (MAK065, Sigma). Cells were seeded at a density of 20,000 cells per well in 96-well plates pre-coated with poly-D-lysine. The next day, the medium was replaced with FBS-free medium (Life Technologies, 11965092). After $1 \mathrm{~h}$ of incubation, the medium was collected and centrifuged at $10,000 \times g$ for $5 \mathrm{~min}$. The secreted L-lactate concentration in the supernatant was colorimetrically determined by an absorbance measurement at $570 \mathrm{~nm}$.

\section{DN-DISC1 mouse model}

To assess the role of DISC1 in energy metabolism in vivo, we utilized an inducible dominant-negative DISC1 (DN-DISC1) model generated using the Tet-off double transgenic (Tg) system as previously described ${ }^{24}$. Briefly, one $\mathrm{Tg}$ line (regulatory line) expresses the tetracycline transactivator (tTA) protein that binds to TetO sequences present on another $\mathrm{Tg}$ line (responder line or tetracycline response element (TRE) line). In our model, mice of the regulatory line are single transgenic GFAP-tTA mice (line 67, a kind gift from Dr. Brian Popko, University of Chicago) that express tTA predominantly in astrocytes ${ }^{25}$. Hemizygous GFAP-tTA mice were crossed with homozygous TRE mice (line 1001) that express C-terminus truncated human protein. The protein product acts as a dominant-negative factor (DN-DISC1) by binding to and decreasing the expression of endogenous mouse DISC1 ${ }^{24}$. Mice of both lines were back-crossed to C57BL/6j background for more than 15 generations. This mating protocol produces litters with $\sim 50 \%$ control mice (TRE-DNDISC1 mice) that do not express DN-DISC1 as they lack tTA and $~ 50 \%$ DN-DISC1 mice that are double Tg mice and express DDN-DISC1 predominantly in astrocytes. Tail tissue samples were used for genotyping as previously described $^{24}$. Developing mice were housed with their dams until postnatal days (P) 21-23, with food and water provided ad libitum. All procedures were approved by the JHU Animal Care and Use Committee.

\section{Indirect calorimetry and energy balance measurements}

To determine whether DN-DISC1 expression in astrocytes affects whole-body metabolism, male DN-DISC1 ( $n$ $=10)$ and control $(n=13)$ mice were monitored individually in an open-circuit indirect calorimeter with additional features to measure ad libitum food intake and physical activity (Comprehensive Lab Animal Monitoring System, CLAMS, Columbus Instruments, Columbus OH). Mice were monitored for four consecutive days, the first three of which involved confirming behavioral and metabolic adaptation. Rates of $\mathrm{O}_{2}$ consumption $\left(\mathrm{VO}_{2}\right)$ and $\mathrm{CO}_{2}$ production $\left(\mathrm{VCO}_{2}\right)$ were measured every $24 \mathrm{~min}$ per mouse and input into software (Oxymax V.4.93) to calculate respiratory exchange ratios $(\mathrm{RER}=\mathrm{VCO} 2 / \mathrm{VO} 2)$ and rates of energy expenditure $(\mathrm{EE})^{26}$. Oxymax calorimetry data outputs were on a per-kg body weight basis, then renormalized to estimate per-kg-lean mass, utilizing body composition data from the mice measured in an EchoMRI-100 immediately prior to CLAMS. Calorimetry data, as well as food intake and physical activity data, are presented in 4-h bins of averaged data per mouse, averaged for each group.

\section{Lactate assay in brain tissue and blood}

We also measured lactate levels in the hippocampus and blood serum of control and DN-DISC1 2-monthold mice. Mice were sacrificed, and their hippocampi and blood samples were collected and processed for lactate measurements according to the manufacturer's method (Lactate Assay Kit II, MAK065, Sigma-Aldrich).

\section{Behavioral tests}

Behavioral tests were performed on 2-month-old control and DN-DISC1 male and female mice. The elevated plus maze (EPM), the forced swim test (FST) and trace fear conditioning (TFC) were used to assess the behavioral effects of preferential L-lactate administration. EPM and FST were described in detail in our previous publications ${ }^{24,27-29}$. TFC was a 3-day test consisting of a habituation day, training day, and test day. Mice were habituated to the shock box (Coulbourn, Holliston, MA) for $10 \mathrm{~min}$. The following day, mice were placed in the shock box, and a 20-s white noise tone was delivered. Twenty seconds following the termination of the tone, a scrambled 2-s $0.5-\mathrm{mA}$ shock was delivered. This tone-shock pairing was repeated three times. On the third day, mice were placed in the shock box for 3 min to measure freezing in response to the context. Following the exposure to the shock box, mice were placed in a different box (a new context not associated with the shock), and the 20-s white noise tone was delivered, during which freezing behavior in response to the tone was measured. 


\section{Lactate treatments}

Mice received single intraperitoneal (ip) injections of vehicle or L-lactate $(1 \mathrm{mg} / \mathrm{kg})$ or D-lactate $(1 \mathrm{mg} / \mathrm{kg}) 1 \mathrm{~h}$ prior to the tests.

\section{Statistical analyses}

Normality and equal variance tests were performed before the following statistical tests were applied to data. Data are expressed as means \pm standard error of the mean (SEM). No statistical methods were used to estimate sample sizes. We determined animal numbers by considering our previous studies on the same mouse model and behavioral tests ${ }^{25}$. All experiments were conducted in triplicate (cell culture and biochemical studies) or with three cohorts of animals (behavioral studies). Mice were randomly allocated to different treatment groups. No animals or data points were excluded from analyses. Although data collection and analysis were not performed with blinding to the experimental conditions, the same analysis and comparison criteria were applied to all cell samples or mice.

OCR and ECAR data were analyzed by two-way repeated analyses of variance (ANOVA). The behavioral and lactate treatment data were analyzed using ANOVA or Student's $t$-test when appropriate. In vitro and biochemical data were analyzed by two-tailed Student's $t$-tests. We used the $F$-test to evaluate the group variance prior to each $t$-test. Bonferroni correction was applied to multiple comparisons; the significance level was set to $p<0.05$.

All raw data are available to the scientific community upon reasonable request.

\section{Results}

No morphological changes in astrocyte mitochondria

We studied the role of DISC1 in energy metabolism in astrocytes using two approaches: DISC1 knockdown (DISC1-KD) (Supplemental Figure S1) and astrocyte selective expression of a dominant-negative, C-terminus truncated human DISC1 (DN-DISC1). Although both endogenous and DN-DISC1 co-localized to mitochondria in primary astrocytes (Supplemental Figure S2-S3), neither DISC1-KD nor DN-DISC1 had any significant effects on the number or shape of mitochondria, including the percentage of abnormal round swollen mitochondria in astrocytes (Supplemental Figure S4). In addition, neither treatment significantly changed the expression of the electron transport chain subunits (Supplemental Fig. 5), the protein import translocase Tim23 (Supplemental Figure 6), or mitofilin, a neuronal DISC1 partner in mitochondria ${ }^{30}$ (Supplemental Figure 7). However, both DISC1-KD and DN-DISC1 significantly decreased the mitochondrial membrane potential, as assessed by TMRE staining (Fig. 1), suggesting abnormal functioning of astrocyte mitochondria produced by altered expression of DISC1.

\section{Abnormal energy metabolism in astrocytes}

To provide a more comprehensive evaluation of the potential role of DISC1 in the energy metabolism in astrocytes, we examined the effects of DISC1-KD and DN-DISC1 on oxidative phosphorylation and oxygen consumption using the Seahorse Mito stress test. Compared with control astrocytes, DISC1-KD or DN-DISC1 significantly decreased basal respiration and carbonyl cyanide m-chlorophenylhydrazone (CCCP)-induced maximal respiration in primary astrocytes (Fig. 2a, b). Somewhat unexpectedly, we also observed a significantly diminished extracellular acidification rate (ECAR) in DISC1-KD and DN-DISC1 primary astrocytes, suggesting declined glycolytic production of lactate (Fig. 2c, d). Prior reports have demonstrated that the oxygen consumption rate and glycolysis rate are two inter-dependent factors that are influenced by oxygen and glucose availability (i.e., the Pasteur and Crabtree effects) ${ }^{31}$. Our results suggest that altered levels of DISC1 might disturb glucose consumption, which in turn could lead to a simultaneous reduction of these two inter-dependent energy pathways in astrocytes. Thus, we hypothesized that chronically reduced uptake of glucose, the key energy substrate, might be responsible for the concurrent decrease in oxidative phosphorylation and glycolysis in DISC1 KD and DN-DISC1 astrocytes.

\section{Diminished glucose uptake and lactate production}

To address this hypothesis, we first measured mRNA expression for the glucose transporters GLUT1 and GLUT4 expressed on astrocytes ${ }^{32}$. We found significantly decreased levels of mRNAs for GLUT4 and significantly increased levels of mRNA for GLUT1 (Supplemental Table 2). We also found significantly reduced protein levels of GLUT4 but not GLUT1 (Fig. 3a-d). Finally, we observed significantly lower glucose uptake by KD-DISC1 or DN-DISC1 astrocytes compared with the uptake observed for control astrocytes (Fig. 3e, f).

As lactate is a leading energy metabolite produced by astrocytes from glucose to support energy demands from neurons during their activity ${ }^{33-35}$, we decided to focus on lactate metabolism to determine the pathophysiological consequences of decreased glucose uptake in the brain. We assessed lactate levels in primary astrocytes and in brain tissues. Compared with control astrocytes, DISC1KD and DN-DISC1 significantly decreased lactate levels in cell pellets and culture medium (Fig. $4 \mathrm{a}-\mathrm{d}$ ). However, no significant effects were observed on expression of the astrocyte-specific monocarboxylate transporter 4 (MCT4), which is responsible for lactate secretion, suggesting that decreased levels of lactate in culture medium were likely due to diminished production of lactate (Supplemental Table 2). We also observed decreased expression of the major enzymes involved in the 


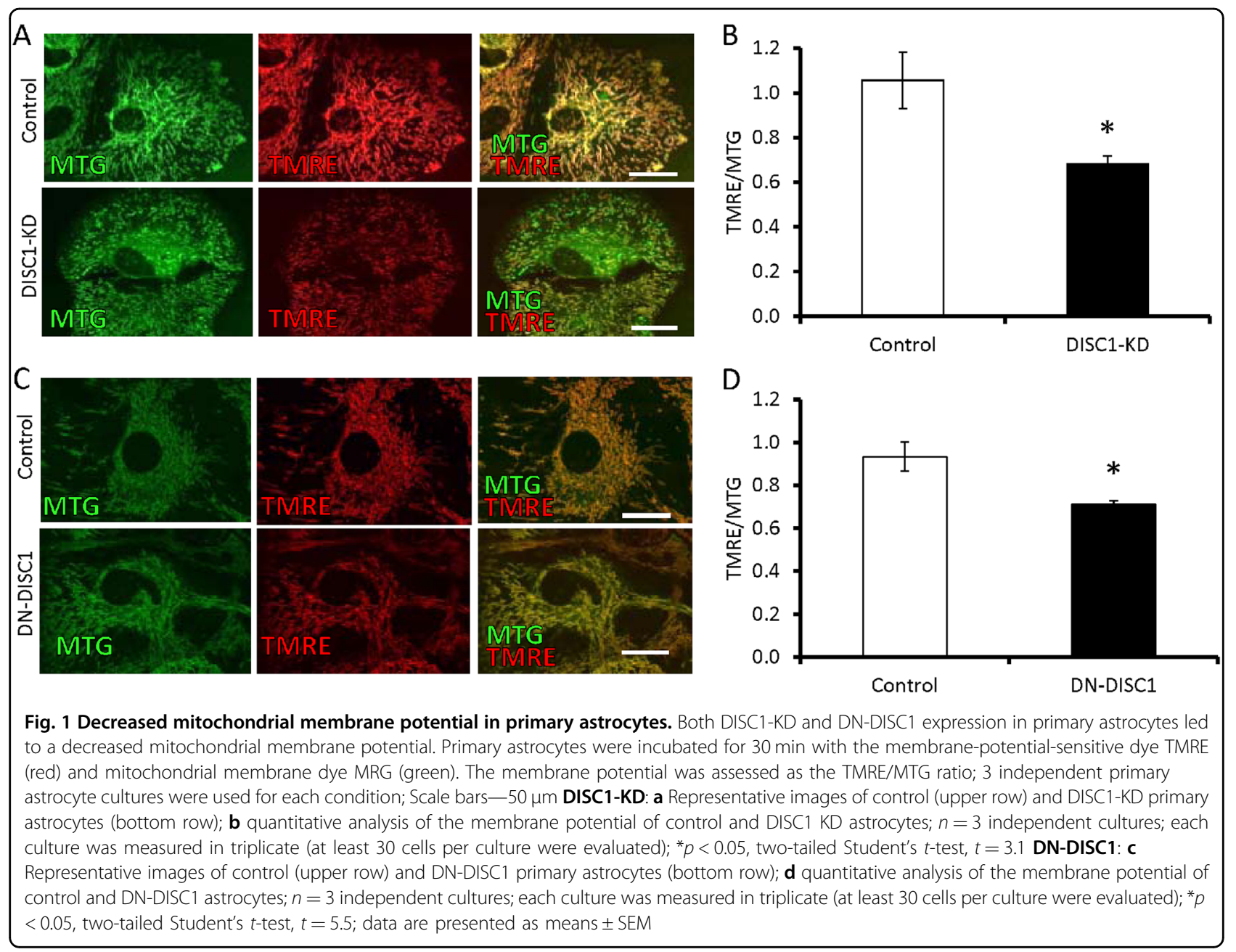

regulation of lactate metabolism in astrocytes (Supplemental Table 2).

Consistent with our in vitro data, we detected significantly lower levels of lactate in the hippocampus of DN-DISC1 mice (Fig. 4e). Notably, we observed no significant group-dependent changes in lactate levels in blood serum (Supplemental Figure 8), suggesting that the diminished concentration of lactate in the hippocampus of DN-DISC1 mice likely results from a reduced production of lactate by brain astrocytes rather than from global changes in lactate metabolism ${ }^{35}$. These results are corroborated by the absence of significant genotyperelated differences in the whole-body energy balance measures, as assessed by the CLAMS experiments (Supplemental Figure 9-11).

\section{Lactate treatment rescues behavioral alterations in DN- DISC1 mice}

Prior reports have demonstrated the role of lactate produced by astrocytes in cognitive function and anxietyand depression-like behaviors in laboratory animals ${ }^{36,37}$.
Thus, we sought to evaluate the effects of lactate treatments on the abnormal behaviors reported by our group for transgenic mice with astrocyte-restricted expression of DN-DISC $1^{38}$. We found that a single acute injection of lactate $(1 \mathrm{mg} / \mathrm{kg}$, ip) significantly decreased anxiety-like behavior in EPM (Fig. 5a, b) and immobility in FST (Fig. $5 \mathrm{c}, \mathrm{d}$ ) and increased cue-dependent freezing in TFC in DN-DISC1 mice. Notably, no significant effects of lactate treatment were observed on locomotor activity in EPM or context-dependent freezing in TFC (Supplemental Figure 12). Furthermore, the enantiomer D-lactate produced no significant effects on the baseline genotype-related differences in these three tests (Supplemental Figures 1314). Collectively, our pharmacology data indicate that acute systemic administration of L-lactate can rescue the behavioral abnormalities in DN-DISC1 mice.

\section{Discussion}

Our findings demonstrate, for the first time, that DISC1 is involved in the regulation of energy metabolism in astrocytes. Both DISC1 knockdown and expression 

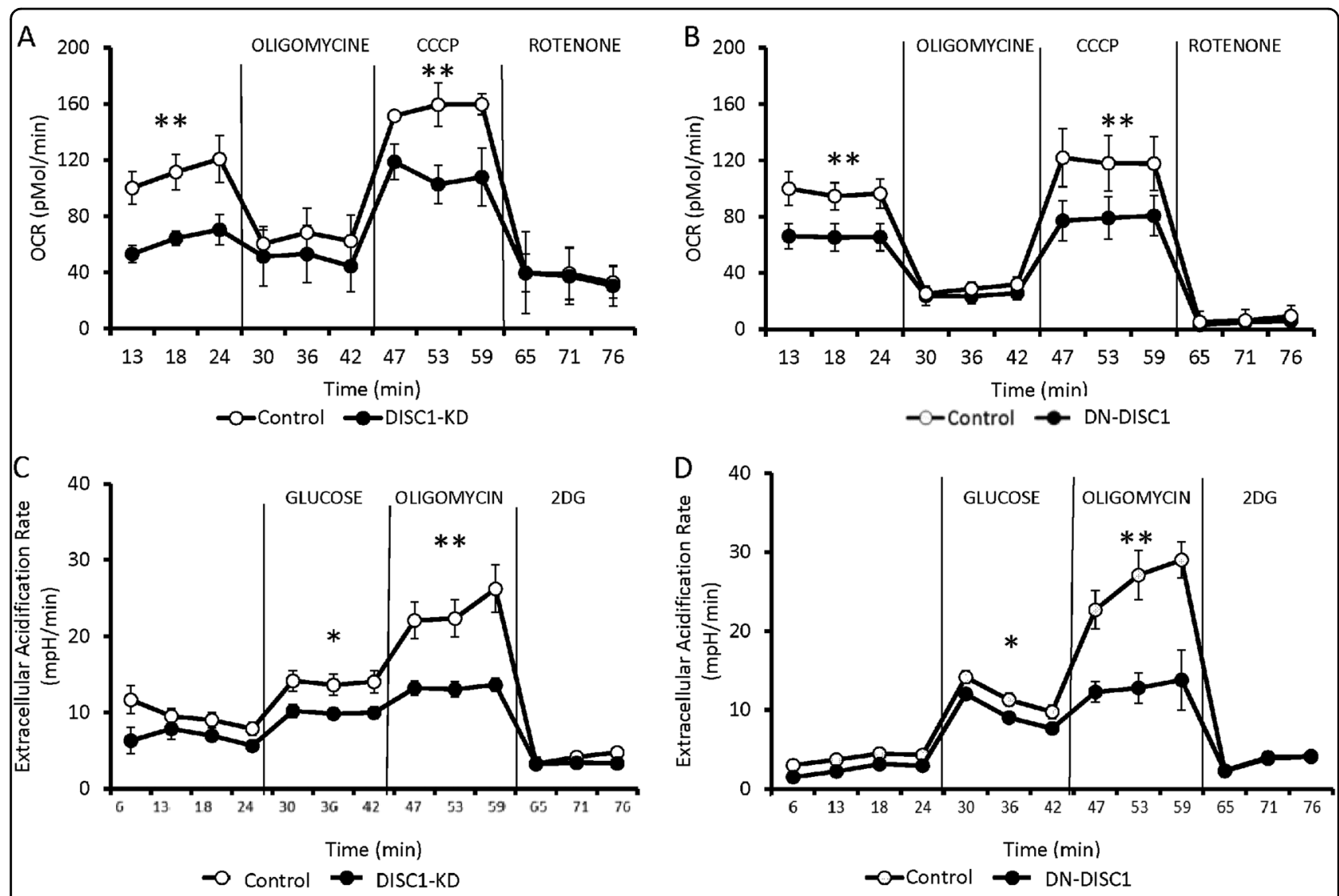

Fig. 2 Metabolic abnormalities in primary astrocytes. Both DISC1-KD and DN-DISC1 inhibited respiration and glycolysis in primary astrocytes. The oxygen consumption rate (OCR) and extracellular acidification rate (ECAR) were measured by Seahorse analysis in primary astrocytes. OCR was measured using the Seahorse XF Cell Mito Stress test after injection of the mitochondrial ETC inhibitors oligomycin (an inhibitor of complex V), CCCP (which uncouples the proton gradient), and rotenone (an inhibitor of complex I). ECAR was measured using the Seahorse XF Glycolysis Stress test after injection of glucose, oligomycin and 2-deoxyglucose (2DG). DISC1-KD: a Representative OCR for control (open circle) and DISC1-KD (solid circle) primary astrocytes; $n=5$ independent cultures; each culture was measured in duplicate, and each experiment was repeated at least three times. Two-way repeated measures ANOVA revealed a significant effect of $K D, F(1,23)=101,69, P<0.01$; a post hoc Bonferroni test showed that basal respiration $(P<0.05)$ and the maximal respiration rate measured after CCCP injection $(P<0.05)$ were significantly lower in DISC1-KD astrocytes than in control astrocytes; ${ }^{*} p<0.05$. b ECAR for control (open circle) and DISC1-KD (solid circle) primary astrocytes; $n=5$ independent cultures; each culture was measured in duplicate, and each experiment was repeated at least three times. Two-way repeated measures ANOVA revealed a significant effect of $\mathrm{KD}, \mathrm{F}(1,23)=175.7, p<0.01$; a post hoc Bonferroni test showed that basal glycolysis $(P<0.05)$ and glycolytic capacity measured after oligomycin injection $(P<0.01)$ were significantly lower in DISC1-KD astrocytes than in control astrocytes; ${ }^{*} p<0.05,{ }^{* *} p<0.01$. DN-DISC1: a Representative OCR for control (open circle) and DN-DISC1 (solid circle) primary astrocytes; $n=5$ independent cultures; each culture was measured in duplicate, and each experiment was repeated at least three times. Two-way repeated measures ANOVA showed a significant effect of DN-DISC1, F(1, 23) $=21.37, P<0.05$; a post hoc Bonferroni test showed that basal respiration $(P<0.05)$ and the maximal respiration rate measured after CCCP injection $(P<0.05)$ were significantly lower in DN-DISC1 astrocytes than in control astrocytes; ${ }^{*} p<0.05$. b ECAR for control (open circle) and DISC1-KD (solid circle) primary astrocytes; $n=5$ independent cultures; each culture was measured in duplicate, and each experiment was repeated at least three times. Two-way repeated measures ANOVA showed a significant effect of DN-DISC1, $F(1,23)=136.11, P<0.001$; a post hoc Bonferroni tests showed that basal glycolysis $(P<0.05)$ and glycolytic capacity measured after oligomycin injection $(P<0.01)$ were significantly lower in DN-DISC1 astrocytes than in control astrocytes; ${ }^{*} p<0.05,{ }^{* *} p<0.01$; data are presented as means \pm SEM

of a dominant-negative DISC1 (DN-DISC1) decreased expression of the glucose transporter 4 (GLUT4), leading to diminished uptake of glucose and decreased production of lactate. These metabolic changes in mice with astrocyte selective expression of DN-DISC1 could contribute to abnormal affective behaviors and deficient spatial memory reversible by systemic lactate treatment.
The present findings significantly expand our knowledge of the roles of DISC1 in different brain cell types with respect to energy metabolism. Prior studies have consistently implicated DISC1 in mitochondrial functions, including mitochondria trafficking, ATP production and $\mathrm{Ca}^{2+}$ buffering ${ }^{39-41}$. However, all reports have focused on neuronal DISC1. As various genetic risk factors could play variable, cell-type-specific roles in the pathogenesis of 

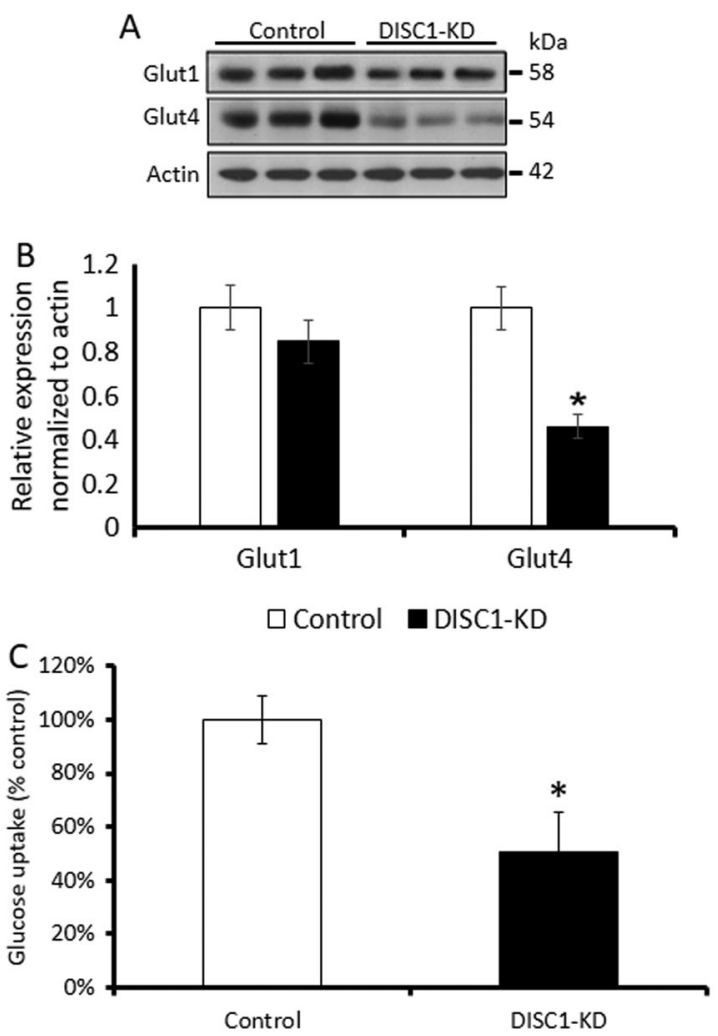
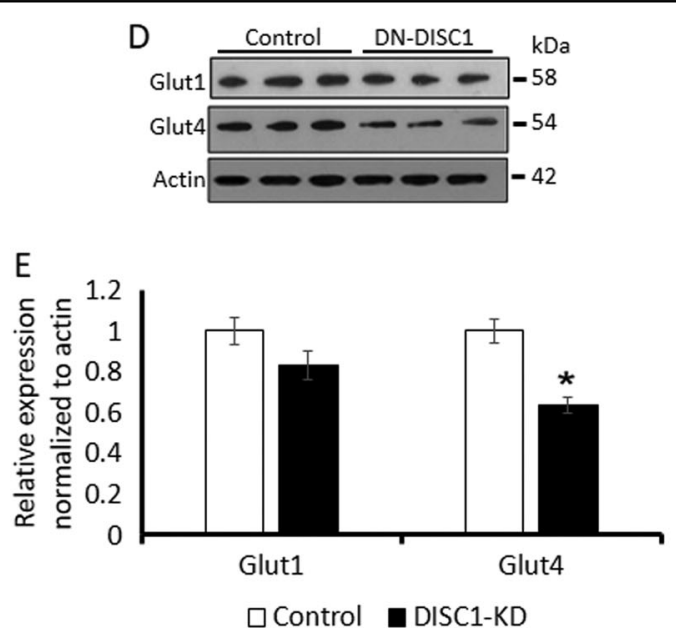

$\mathrm{F}$

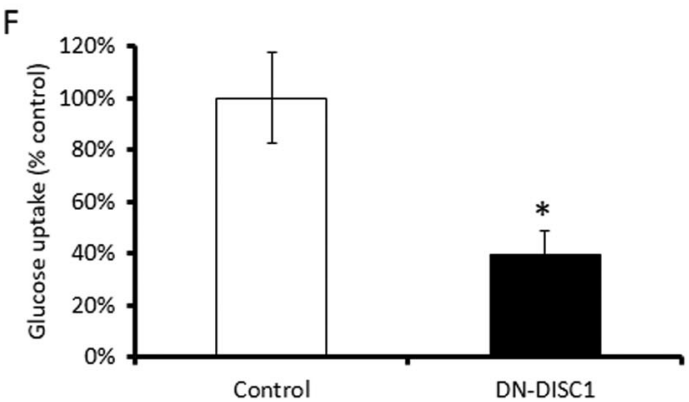

Fig. 3 Decreased expression of GLUT4 and glucose uptake by primary astrocytes. Expression of the glucose transporters and glucose uptake were measured in primary astrocytes. Both DISC1-KD and DN-DISC1 decreased expression of GLUT4 and glucose uptake in primary astrocytes. DISC1KD: a Representative Western blotting images of expression of the glucose transporters by control and DISC1-KD primary astrocytes; $\mathbf{b}$ quantitative analysis of the expression of the transporters by control (open bars) and DISC1-KD (solid bars) astrocytes; $n=3$ independent cultures; each culture was measured in triplicate; ${ }^{*} p<0.05$, two-tailed Student's $t$-test; $t=-4.808$ for GLUT4; c quantitative analysis of glucose uptake by control (open bars) and DISC1-KD (solid bars) astrocytes; $n=4$ independent cultures; each culture was measured in triplicate; ${ }^{*} p<0.05$, two-tailed Student's $t$-test, $t=$ 3.254. DN-DISC1: $\mathbf{d}$ Representative Western blotting images of expression of the glucose transporters by control (open bars) and DN-DISC1 (solid bars) primary astrocytes; e quantitative analysis of expression of the glucose transporters by control (open bars) and DN-DISC1 (solid bars) primary astrocytes; $n=3$ independent cultures; each culture was measured in triplicate; ${ }^{*} p<0.05$, two-tailed Student's $t$-test; $t=-4.673$ for GLUT4; $\mathbf{f}$ quantitative analysis of glucose uptake by control (open bars) and DN-DISC1 (solid bars) astrocytes; $n=4$ independent cultures; each culture was measured in triplicate; * $p<0.05$, two-tailed Student's $t$-test, $t=3.049$; data are presented as means \pm SEM

psychiatric disorders ${ }^{20,42}$, it is important that we elucidate the contribution of genetic variants expressed in different brain cells. In this context, our work provides new insights into the possible role of DISC1 in energy metabolism in astrocytes. Our findings suggest that altered DISC1 expression in astrocytes affects glucose uptake, which leads to diminished lactate production, one of the major energy supply processes in astrocytes ${ }^{35,43,44}$. Because both DISC1-KD and DN-DISC1 approaches to manipulating DISC1 expression in astrocytes produced comparable outcomes, we believe that potential off-target effects of either manipulation are less likely, suggesting that the present study points to the critical role of DISC1 in energy metabolism in astrocytes.

Although the current study does not directly address the mechanisms of how DISC1 is involved in glucose uptake regulation, one could propose that, as a scaffolding $\operatorname{protein}^{45}$, DISC1 is involved in trafficking and/or membrane recycling of glucose transporters ${ }^{46}$. Astrocytes express two glucose transporters, GLUT1 and GLUT4. Compared with that of GLUT1, expression of GLUT4 in astrocytes is 20 times lower ${ }^{47}$. GLUT4 is an insulinsensitive glucose transporter that is translocated to the cell membrane when insulin activates PI3K/Akt signaling $^{48,49}$. GLUT4 is highly expressed in the hippocampus, olfactory bulbs and cerebellum ${ }^{50,51}$. Although the role of insulin signaling in the brain is poorly understood, previous studies have suggested that hippocampal GLUT4 could play a key role in learning and memory ${ }^{52}$. Notably, we found that astrocyte-specific expression of DN-DISC1 decreased levels of GLUT4 in the hippocampus and impaired performance in trace fear conditioning.

Unlike GLUT4 and despite decreased glucose uptake, we found no significant changes in protein levels of 


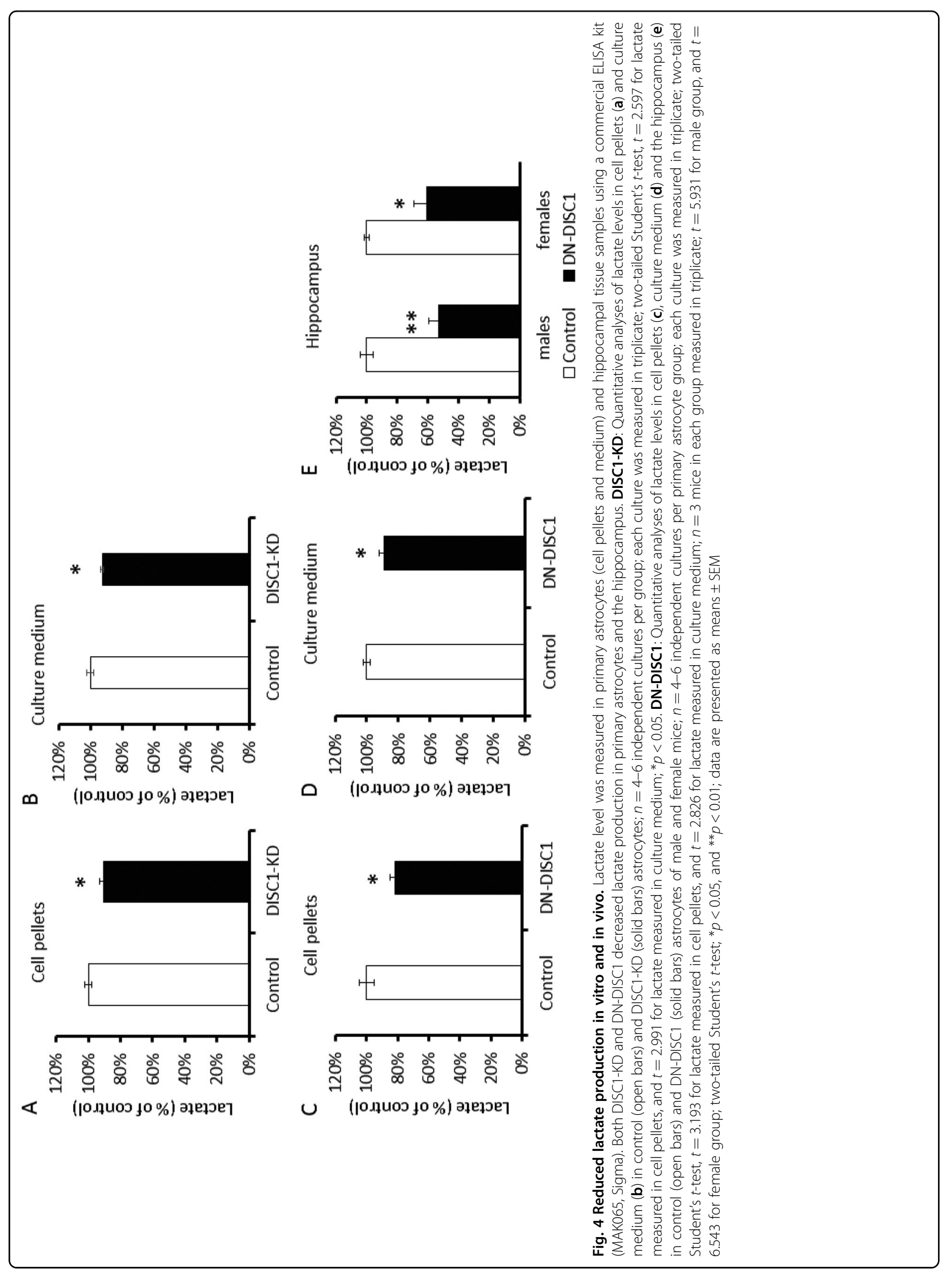



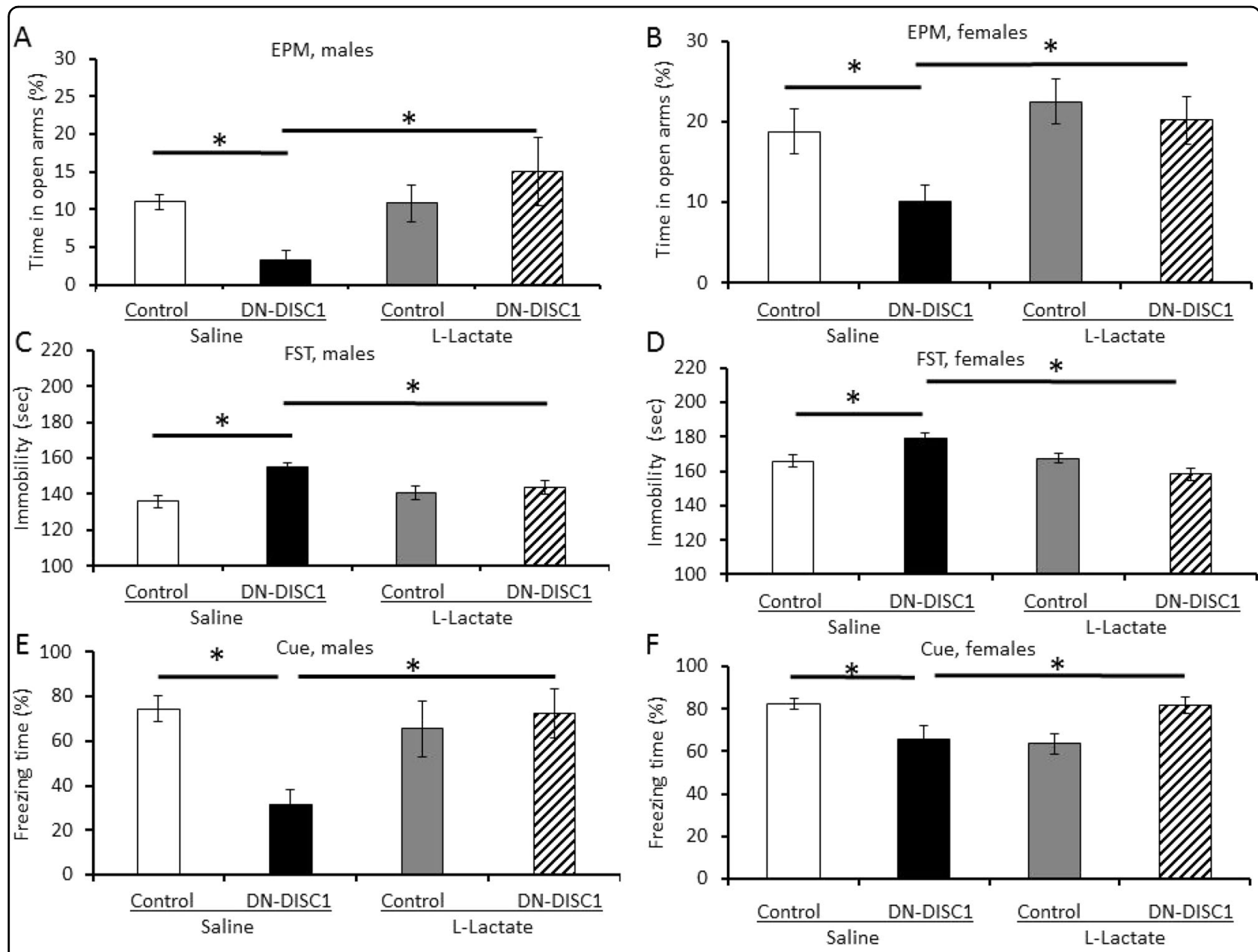

Fig. 5 L-lactate administration rescues the behavioral changes in mice. Control and DN-DISC1 male and female 2-month-old mice received a single injection of L-lactate at a dose of $1 \mathrm{mg} / \mathrm{kg}$ (IP) 60 min before behavioral testing. Lactate treatment rescued the behavioral abnormalities in DNDISC1 mice. EPM: Lactate administration significantly increased time spent in open arms of the EPM in DN-DISC1 male $(\mathbf{a})$ and female $(\mathbf{b})$ mice; $n=$ 4-6 male and 6-11 female mice in each group. For male mice, two-way ANOVA showed a significant genotype $\times$ treatment interaction, $F(1,22)=$ 5.197, $p=0.036$. A post hoc Bonferroni test showed that compared with saline-treated control mice, saline-treated DN-DISC1 mice spent significantly less time in open arms of the EPM $(p=0.026)$, and compared with saline-treated DN-DISC1 mice, lactate-treated DN-DISC1 mice spent significantly more time in open arms of the EPM $(p=0.022)$. For female mice, two-way ANOVA showed a significant effect of treatment, $F(1,29)=5.532, p=$ 0.026; a post hoc Bonferroni $t$-test showed that compared with saline-treated control mice, saline-treated DN-DISC1 mice spent significantly less time in open arms of the EPM ( $p=0.047)$, and compared with saline-treated DN-DISC1 mice, lactate-treated DN-DISC1 mice spent significantly more time in open arms of the EPM $(p=0.007)$. FST: Lactate administration significantly decreased immobility in FST in DN-DISC1 male (c) and female (d) mice; $n=6$ male and $n=7-11$ female mice in each group. For male mice, two-way ANOVA showed a significant effect of genotype, $\mathrm{F}(1,22)=11.00, p=$ 0.003 , and a significant genotype by treatment interaction, $F(1,22)=5.64, p=0.027$. A post hoc Bonferroni test showed that compared with salinetreated control mice, saline-treated DN-DISC1 mice exhibited significantly greater immobility $(p<0.001)$, and compared with saline-treated DN-DISC1 mice, lactate-treated DN-DISC1 mice showed significantly less immobility in the FST $(p=0.032)$. For female mice, two-way ANOVA showed a significant effect of treatment, $F(1,28)=6.33, p=0.018$, and a genotype by treatment interaction, $F(1,28)=8.61, p=0.007$. A post hoc Bonferroni test showed that compared with saline-treated control mice, saline-treated DN-DISC1 mice exhibited significantly greater immobility $(p=0.041)$, and compared with saline-treated DN-DISC1 mice, lactate-treated DN-DISC1 mice showed significantly less immobility in the FST ( $p=0.001)$. TFC: Lactate administration significantly increased cue-dependent freezing in TFC in DN-DISC1 male (e) and female (f) mice; $n=4-6$ male and 7-15 female mice in each group. For male mice, two-way ANOVA showed a significant genotype by treatment interaction, $F(1,17)=6.37, p=0.022$. A post hoc Bonferroni test showed that compared with saline-treated control mice, saline-treated DN-DISC1 exhibited significantly less cue-dependent freezing $(p<0.006)$, and compared with saline-treated DN-DISC1 mice, lactate-treated DN-DISC1 mice showed significantly more cue-dependent freezing ( $p$ $=0.009$ ). For female mice, two-way ANOVA showed a significant genotype by treatment interaction, $\mathrm{F}(1,43)=10.73, p=0.002$. A post hoc Bonferroni test showed that compared with saline-treated control mice, saline-treated DN-DISC1 mice exhibited significantly less cue-dependent freezing $(p=0.044)$, and compared with saline-treated DN-DISC1 mice, lactate-treated DN-DISC1 mice showed significantly more cue-dependent freezing $(p=0.016)$; data are presented as means \pm SEM 
GLUT1. However, given the major role of GLUT1 in glucose uptake in astrocytes, the reason for the inconsistency between unaltered GLUT1 levels and reduced glucose uptake remains unclear. It is possible that impaired trafficking of GLUT1 in DISC1-KD and DNDISC1 astrocytes could lead to comparable levels of GLUT1, as assessed by Western blotting and reduced glucose uptake. For example, DISC1-KD has been reported to decrease activation of $\mathrm{PI}^{3} \mathrm{~K}^{53}$, which regulates intracellular transport of GLUT1 to the plasma membrane $^{54-59}$. In addition, GLUT1 intracellular transport could be impaired due to abnormal DISC1-mediated activation of RHOA/ROCK $1^{60}$ and/or RAS ${ }^{57,61,62}$. Thus, it is tempting to speculate that DISC1-KD and DN-DISC1 may have affected intracellular trafficking of GLUT1, leading to accumulation of the transporter in the cytoplasm and an ensuing decrease in glucose uptake. Increased expression of Slc2a1 could be a compensatory response. Similarly, consistent with decreased glucose uptake, Ldha (lactate dehydrogenase A), Ldhb (lactate dehydrogenase B), and Pgk1 (phosphoglycerate kinase) expression is a compensatory response to diminished glucose uptake by primary astrocytes. Future studies will address the putative molecular mechanisms whereby DISC1 regulates glucose uptake.

Several lines of evidence suggest that brain bioenergetics are altered in schizophrenia and bipolar disorder ${ }^{63}$. Multiple reports have indicated reduced energy metabolism in the dorsolateral prefrontal cortex (DLPFC) in schizophrenia ${ }^{64}$, alterations in glucose homeostasis at the onset of the illness ${ }^{65,66}$, and impaired glucose tolerance in first-episode psychosis patients ${ }^{67,68}$. However, negative findings have also been reported ${ }^{69}$. Taken together, these results suggest that abnormal glucose metabolism may be associated with the pathogenesis and psychopathology of schizophrenia in the early phases of the disease ${ }^{70}$. Studies of patients with bipolar disorder have also found decreased high-energy phosphates, suggesting a shift toward glycolysis for energy production ${ }^{71}$, impaired oxidative phosphorylation and decreased total energy production and/or substrate availability ${ }^{72}$. Although we are not aware of any reports on metabolic abnormalities in members of the Scottish translocation family, our findings warrant future studies of this possibility.

The data indicate that a loss of function of DISC1 and resulting decreased lactate production could contribute to the behavioral changes observed in DN-DISC1 mice. Our results are consistent with growing evidence indicating that astrocytes play a critical role in energy supply traffic by up-taking glucose and producing lactate via glycolysis or glycogenolysis ${ }^{40}$. Notably, the astrocyte-neuron lactate shuttle hypothesis is not inconsistent with the fact that glucose also represents a major energy substrate in the brain and both metabolites are critical for proper brain functioning ${ }^{73}$. Our research is in line with a number of studies that demonstrate that brain lactate may influence learning and memory by maintaining an appropriate energy supply level during cognitive processing ${ }^{74}$. For example, direct intrahippocampal injections of lactate rescue working memory ${ }^{74}$ and passive avoidance training $^{35}$. Pharmacological or genetic suppression of lactate transport in the brain inhibits long-term memory and decreases the rescue ability of lactate ${ }^{35,74,75}$. Our data are also in agreement with recent studies demonstrating that peripheral administration of lactate had antidepressantlike effects similar to those induced by desipramine ${ }^{33}$. We found that systemic acute injections of lactate increased time spent by DN-DISC1 mice in open arms of the EPM and decreased immobility time in the FST.

Our study does not address the regional effects of lactate metabolism on mouse behaviors. Although we assessed hippocampus-dependent behaviors, we do not suggest that this brain region exclusively mediates the behavioral abnormalities associated with DISC1 manipulations and/or pharmacological effects of lactate. Future studies will address these possibilities using regional genetic manipulation and/or lactate injections. We did not evaluate the potential neuronal changes in mice with reduced lactate production. Given that acute lactate treatment ameliorated the behavioral changes in DNDISC1 mice, it is tempting to speculate that putative neuronal abnormalities are likely.

In conclusion, the present study demonstrates, for the first time, that DISC1 may be involved in the regulation of energy metabolism in astrocytes to support neuronal activity and associated behaviors. Altered expression of DISC1 in astrocytes leads to decreased glucose uptake and reduction of lactate production that could be responsible for affective and cognitive disorders consistent with aspects of major mental illnesses.

\footnotetext{
Acknowledgements

This work was supported by MH-083728, MH-094268 the Conte Center grant, and The Brain and Behavior Research Foundation (MVP), DA00266 and NSR37 NS067525 (TMD and VLD). The authors also thank Mr. Glen Hatfield for his expert help with qRT-PCR experiments. Funding:The authors declare no biomedical financial interests or potential conflicts of interest.
}

\footnotetext{
Author details

${ }^{1}$ Departments of Psychiatry and Behavioral Sciences, Johns Hopkins University School of Medicine, Baltimore, MD 21287, USA. ²Departments of Pathology, Johns Hopkins University School of Medicine, Baltimore, MD 21287, USA. ${ }^{3}$ Depart ment of Pharmacology \& Toxicology, University of Alabama at Birmingham School of Medicine, Birmingham, AL 35294, USA. ${ }^{4}$ Stanley Neurovirology Laboratory, Department of Pediatrics, Johns Hopkins University School of Medicine, Baltimore, MD 21287, USA. ${ }^{5}$ Departments of Neurology, Johns Hopkins University School of Medicine, Baltimore, MD 21287, USA. ${ }^{6}$ Departments of Physiology, Johns Hopkins University School of Medicine, Baltimore, MD 21287, USA. ${ }^{7}$ Solomon H. Snyder Department of Neuroscience, Johns Hopkins University School of Medicine, Baltimore, MD 21287, USA. ${ }^{8}$ Neuroregeneration and Stem Cell Programs, Institute for Cell Engineering, Johns Hopkins University School of Medicine, Baltimore, MD 21205, USA. ${ }^{9}$ Departments of Pharmacology and Molecular Sciences, Johns Hopkins
} 
University School of Medicine, Baltimore, MD 21287, USA. ${ }^{10} \mathrm{Center}$ for Metabolic and Obesity Research, Johns Hopkins University School of Medicine, Baltimore, MD 21205, USA. "'Departments of Cell Biology, Johns Hopkins University School of Medicine, Baltimore, MD 21287, USA. ${ }^{12}$ Department of Neuroscience, Novosibirsk State University, Novosibirsk, Russia. ${ }^{13}$ Department of Experimental \& Clinical Neuroscience, Institute of Physiology \& Basic Medicine, Novosibirsk 630090, Russian Federation

\section{Conflict of interest}

The authors declare that they have no conflict of interest.

\section{Publisher's note}

Springer Nature remains neutral with regard to jurisdictional claims in published maps and institutional affiliations.

Supplementary Information accompanies this paper at https://doi.org/ 10.1038/s41398-018-0123-9.

Received: 10 July 2017 Revised: 11 January 2018 Accepted: 18 February 2018 Published online: 12 April 2018

\section{References}

1. Chung, W. S., Welsh, C. A., Barres, B. A. \& Stevens, B. Do glia drive synaptic and cognitive impairment in disease? Nat. Neurosci. 18, 1539-1545 (2015).

2. Molofsky, A. V. et al. Astrocytes and disease: a neurodevelopmental perspective. Genes Dev. 26, 891-907 (2012).

3. Bushong, E. A., Martone, M. E., Jones, Y. Z. \& Ellisman, M. H. Protoplasmic astrocytes in CA1 stratum radiatum occupy separate anatomical domains. J. Neurosci. 22, 183-192 (2002).

4. Rossi, D. \& Volterra, A. Astrocytic dysfunction: insights on the role in neurodegeneration. Brain Res. Bull. 80, 224-232 (2009).

5. Chechik, T., Roeder, L. M., Tildon, J. T. \& Poduslo, S. E. Ketone body enzyme activities in purified neurons, astrocytes and oligodendroglia. Neurochem. Int. 10, 95-99 (1987).

6. Sullivan, P. F., Daly, M. J. \& O'Donovan, M. Genetic architectures of psychiatric disorders: the emerging picture and its implications. Nat. Rev. Genet. 13, 537-551 (2012).

7. Kondziella, D., Brenner, E., Eyjolfsson, E. M. \& Sonnewald, U. How do glialneuronal interactions fit into current neurotransmitter hypotheses of schizophrenia? Neurochem. Int. 50, 291-301 (2007).

8. Schnieder, T. P. \& Dwork, A. J. Searching for neuropathology: gliosis in schizophrenia. Biol. Psychiatry 69, 134-139 (2011).

9. Millar, J. K. et al. Genomic structure and localisation within a linkage hotspot of disrupted in Schizophrenia 1, a gene disrupted by a translocation segregating with schizophrenia. Mol. Psychiatry 6, 173-178 (2001).

10. Schizophrenia Working Group of the Psychiatric Genomics C. Biological insights from 108 schizophrenia-associated genetic loci. Nature 511, 421-427 (2014).

11. Farrell, M. S. et al. Evaluating historical candidate genes for schizophrenia. Mol. Psychiatry 20, 555-562 (2015).

12. Ji, B. et al. Inhibition of protein translation by the DISC1-Boymaw fusion gene from a Scottish family with major psychiatric disorders. Hum. Mol. Genet. 23 5683-5705 (2014).

13. Millar, J. K., James, R., Christie, S. \& Porteous, D. J. Disrupted in schizophrenia 1 (DISC1): subcellular targeting and induction of ring mitochondria. Mol. Cell. Neurosci. 30, 477-484 (2005).

14. James, R. et al. Disrupted in Schizophrenia 1 (DISC1) is a multicompartmentalized protein that predominantly localizes to mitochondria. Mol. Cell. Neurosci. 26, 112-122 (2004).

15. Norkett, R. et al. DISC1-dependent regulation of mitochondrial dynamics controls the morphogenesis of complex neuronal dendrites. J. Biol. Chem. 291, 613-629 (2016)

16. Ogawa, F. et al. DISC1 complexes with TRAK1 and Miro1 to modulate anterograde axonal mitochondrial trafficking. Hum. Mol. Genet. 23, 906-919 (2014).

17. Pinero-Martos, E. et al. Disrupted in schizophrenia 1 (DISC1) is a constituent of the mammalian mitochondrial contact site and cristae organizing system
(MICOS) complex, and is essential for oxidative phosphorylation. Hum. Mol. Genet. 25, 4157-4169 (2016).

18. Eykelenboom, J. E. et al. A t $(1 ; 11)$ translocation linked to schizophrenia and affective disorders gives rise to aberrant chimeric DISC1 transcripts that encode structurally altered, deleterious mitochondrial proteins. Hum. Mol. Genet. 21, 3374-3386 (2012).

19. Park, C. et al. Disrupted-in-schizophrenia 1 (DISC1) and Syntaphilin collaborate to modulate axonal mitochondrial anchoring. Mol. Brain 9, 69 (2016).

20. Xia, M. et al. Cell type-specific effects of mutant DISC1: a proteomics study. Mol. Neuropsychiatry 2, 28-36 (2016).

21. Gao, W., Pu, Y., Luo, K. Q. \& Chang, D. C. Temporal relationship between cytochrome $c$ release and mitochondrial swelling during UV-induced apoptosis in living HeLa cells. J. Cell. Sci. 114, 2855-2862 (2001). Pt 15.

22. Cooper, O. et al. Pharmacological rescue of mitochondrial deficits in iPSCderived neural cells from patients with familial Parkinson's disease. Sci. Transl. Med. 4, 141ra190 (2012).

23. Abe, Y., Sakairi, T., Beeson, C. \& Kopp, J. B. TGF-beta1 stimulates mitochondrial oxidative phosphorylation and generation of reactive oxygen species in cultured mouse podocytes, mediated in part by the mTOR pathway. Am. J. Physiol. Ren. Physiol. 305, F1477-F1490 (2013).

24. Pletnikov, M. V. et al. Inducible expression of mutant human DISC1 in mice is associated with brain and behavioral abnormalities reminiscent of schizophrenia. Mol. Psychiatry 13, 173-186 (2008). 115.

25. Terrillion, C. E. et al. DISC1 in astrocytes influences adult neurogenesis and hippocampus-dependent behaviors in mice. Neuropsychopharmacology $\mathbf{4 2}$, 2242-2251 (2017)

26. Lusk, G. The elements of the science of nutrition. 4th ed, (Academic Press, New York, NY, 1976). vol. Reprint, New York: Johnson Reprint Corp.

27. Abazyan, B. et al. Chronic exposure of mutant DISC1 mice to lead produces sex-dependent abnormalities consistent with schizophrenia and related mental disorders: a gene-environment interaction study. Schizophr. Bull. 40, 575-584 (2014).

28. Abazyan, B. et al. Prenatal interaction of mutant DISC1 and immune activation produces adult psychopathology. Biol. Psychiatry 68, 1172-1181 (2010).

29. Ayhan, Y. et al. Differential effects of prenatal and postnatal expressions of mutant human DISC1 on neurobehavioral phenotypes in transgenic mice: evidence for neurodevelopmental origin of major psychiatric disorders. Mol. Psychiatry 16, 293-306 (2011).

30. Park, Y. U. et al. Disrupted-in-schizophrenia 1 (DISC1) plays essential roles in mitochondria in collaboration with Mitofilin. Proc. Natl Acad. Sci. USA 107, 17785-17790 (2010).

31. Krebs, H. A. The Pasteur effect and the relations between respiration and fermentation. Essays Biochem. 8, 1-34 (1972).

32. Falkowska, A. et al. Energy metabolism of the brain, including the cooperation between astrocytes and neurons, especially in the context of glycogen metabolism. Int. J. Mol. Sci. 16, 25959-25981 (2015).

33. Carrard A. et al. Peripheral administration of lactate produces antidepressantlike effects. Mol. Psychiatry 23: 392-399 (2016).

34. Machler, P. et al. In vivo evidence for a lactate gradient from astrocytes to neurons. Cell Metab. 23, 94-102 (2016).

35. Suzuki, A. et al. Astrocyte-neuron lactate transport is required for long-term memory formation. Cell 144, 810-823 (2011).

36. Gibbs, M. E., Anderson, D. G. \& Hertz, L. Inhibition of glycogenolysis in astrocytes interrupts memory consolidation in young chickens. Glia 54, 214-222 (2006).

37. Gilmartin, M. R. \& Helmstetter, F. J. Trace and contextual fear conditioning require neural activity and NMDA receptor-dependent transmission in the medial prefrontal cortex. Learn. Mem. 17, 289-296 (2010).

38. Terrillion, C. E. et al. DISC1 in astrocytes influences adult neurogenesis and hippocampus-dependent behaviors in mice. Neuropsychopharmacology $\mathbf{4 2}$ 2242-2251 (2017)

39. Murphy, L. C. \& Millar, J. K. Regulation of mitochondrial dynamics by DISC1, a putative risk factor for major mental illness. Schizophr. Res. 187, 55-61 (2017).

40. Norkett, R., Modi, S. \& Kittler, J. T. Mitochondrial roles of the psychiatric disease risk factor DISC1. Schizophr. Res. 187, 47-54 (2017).

41. Park, C. \& Park, S. K. Molecular links between mitochondrial dysfunctions and schizophrenia. Mol. Cells 33, 105-110 (2012).

42. Xia, M., Abazyan, S., Jouroukhin, Y. \& Pletnikov, M. Behavioral sequelae of astrocyte dysfunction: focus on animal models of schizophrenia. Schizophr. Res. 176, 72-82 (2016). 
43. Dienel, G. A. \& Hertz, L. Glucose and lactate metabolism during brain activation. J. Neurosci. Res. 66, 824-838 (2001).

44. Magistretti, P. J. Neuron-glia metabolic coupling and plasticity. Exp. Physiol. 96, 407-410 (2011)

45. Hayashi-Takagi, A. et al. Disrupted-in-Schizophrenia 1 (DISC1) regulates spines of the glutamate synapse via Rac1. Nat. Neurosci. 13, 327-332 (2010).

46. Leto, D. \& Saltiel, A. R. Regulation of glucose transport by insulin: traffic control of GLUT4. Nat. Rev. Mol. Cell. Biol. 13, 383-396 (2012).

47. Zhang, Y. et al. An RNA-sequencing transcriptome and splicing database of glia, neurons, and vascular cells of the cerebral cortex. J. Neurosci. 34 11929-11947 (2014).

48. McEwen, B. S. \& Reagan, L. P. Glucose transporter expression in the central nervous system: relationship to synaptic function. Eur. J. Pharmacol. 490, 13-24 (2004).

49. Reagan, L. P. Neuronal insulin signal transduction mechanisms in diabetes phenotypes. Neurobiol. Aging 26, 56-59 (2005).

50. El Messari, S., Ait-lkhlef, A., Ambroise, D. H., Penicaud, L. \& Arluison, M. Expression of insulin-responsive glucose transporter GLUT4 mRNA in the rat brain and spinal cord: an in situ hybridization study. J. Chem. Neuroanat. 24 225-242 (2002)

51. Vannucci, S. J. et al. GLUT4 glucose transporter expression in rodent brain: effect of diabetes. Brain Res. 797, 1-11 (1998).

52. Pearson-Leary, J. \& McNay, E. C. Novel roles for the insulin-regulated glucose transporter-4 in hippocampally dependent memory. J. Neurosci. 36 11851-11864 (2016).

53. Rodriquez-Seoane, C., Ramos, A., Korth, C. \& Requena, J. R. DISC1 regulates expression of the neurotrophin VGF through the PI3KAKT/CREB pathway. J. Neurochem. 135, 598-605 (2015).

54. Siska, P. J. \& Rathmell, J. C. PKCs sweeten cell metabolism by phosphorylation of Glut1. Mol. Cell 58, 711-712 (2015).

55. Wieman, H. L., Wofford, J. A. \& Rathmell, J. C. Cytokine stimulation promotes glucose uptake via phosphatidylinositol-3 kinase/Akt regulation of Glut1 activity and trafficking. Mol. Biol. Cell 18, 1437-1446 (2007).

56. Kaliman, P., Vinals, F., Testar, X., Palacin, M. \& Zorzano, A. Disruption of GLUT1 glucose carrier trafficking in L6E9 and Sol8 myoblasts by the phosphatidylinositol 3-kinase inhibitor wortmannin. Biochem. J. 312, 471-477 (1995).

57. Makinoshima, H. et al. Signaling through the phosphatidylinositol 3-kinase (PI3K)/Mammalian target of rapamycin (mTOR) axis is responsible for aerobic glycolysis mediated by glucose transporter in epidermal growth factor receptor (EGFR)-mutated lung adenocarcinoma. J. Biol. Chem. 290 17495-17504 (2015)

58. Morani, F. et al. PTEN regulates plasma membrane expression of glucose transporter 1 and glucose uptake in thyroid cancer cells. J. Mol. Endocrinol. 53, 247-258 (2014).
59. Palmada, M. et al. SGK1 kinase upregulates GLUT1 activity and plasma membrane expression. Diabetes 55, 421-427 (2006).

60. Gao, X. et al. Disrupted in schizophrenia 1 (DISC1) inhibits glioblastoma development by regulating mitochondria dynamics. Oncotarget 7, 85963-85974 (2016)

61. Wang, S. et al. DISC1 regulates astrogenesis in the embryonic brain via modulation of RAS/MEK/ERK signaling through RASSF7. Development 143, 2732-2740 (2016)

62. Kozma, L. et al. The ras signaling pathway mimics insulin action on glucose transporter translocation. Proc. Natl Acad. Sci. USA 90, 4460-4464 (1993).

63. Dean, B., Thomas, N., Scarr, E. \& Udawela, M. Evidence for impaired glucose metabolism in the striatum, obtained postmortem, from some subjects with schizophrenia. Transl. Psychiatry 6, e949 (2016).

64. Pinacho, R. et al. The glial phosphorylase of glycogen isoform is reduced in the dorsolateral prefrontal cortex in chronic schizophrenia. Schizophr. Res. 177, 37-43 (2016).

65. Ryan, M. C., Collins, P. \& Thakore, J. H. Impaired fasting glucose tolerance in first-episode, drug-naive patients with schizophrenia. Am. J. Psychiatry 160 284-289 (2003).

66. Pillinger, T. et al. Impaired glucose homeostasis in first-episode schizophrenia: a systematic review and meta-analysis. JAMA Psychiatry 74, 261-269 (2017).

67. Petrikis, P. et al. Parameters of glucose and lipid metabolism at the fasted state in drug-naive first-episode patients with psychosis: evidence for insulin resistance. Psychiatry Res. 229, 901-904 (2015).

68. Perry, B. I., McIntosh, G., Weich, S., Singh, S. \& Rees, K. The association between first-episode psychosis and abnormal glycaemic control: systematic review and meta-analysis. Lancet Psychiatry 3, 1049-1058 (2016).

69. Sengupta, S. et al. Are metabolic indices different between drug-naive firstepisode psychosis patients and healthy controls? Schizophr. Res. 102, 329-336 (2008).

70. Zhang, X. Y. et al. Glucose disturbances in first-episode drug-naive schizophrenia: relationship to psychopathology. Psychoneuroendocrinology $6 \mathbf{2}$ 376-380 (2015).

71. Callaly, E. et al. Mitochondrial dysfunction in the pathophysiology of bipolar disorder: effects of pharmacotherapy. Mini. Rev. Med. Chem. 15, 355-365 (2015).

72. Stork, C. \& Renshaw, P. F. Mitochondrial dysfunction in bipolar disorder: evidence from magnetic resonance spectroscopy research. Mol. Psychiatry 10 900-919 (2005).

73. Magistretti, P. in Fundamental Neuroscience (eds Squire, B. D. L. R. et al.) 271-293 (Academic Press, San Diego, 2008).

74. Newman, L. A., Korol, D. L. \& Gold, P. E. Lactate produced by glycogenolysis in astrocytes regulates memory processing. PLoS One 6, e28427 (2011).

75. Zhang, Y. et al. Inhibition of lactate transport erases drug memory and prevents drug relapse. Biol. Psychiatry 79, 928-939 (2016). 